\title{
Multitarget, selective compound design yields picomolar inhibitors of a kinetoplastid pteridine reductase 1
}

Ina Pöhner ${ }^{1,2 \dagger}$, Antonio Quotadamo ${ }^{3,4 \dagger}$, Joanna Panecka-Hofman ${ }^{1,5}$, Rosaria Luciani ${ }^{6}$, Matteo Santucci ${ }^{6}$, Pasquale Linciano ${ }^{6}$, Giacomo Landi ${ }^{7}$, Flavio Di Pisa ${ }^{7}$, Lucia Dello lacono ${ }^{7}$, Cecilia Pozzi $^{7}$, Stefano Mangani ${ }^{7}$, Sheraz $\mathrm{Gul}^{8}$, Gesa Witt ${ }^{8}$, Bernhard Ellinger ${ }^{8}$, Maria Kuzikov ${ }^{8}$, Nuno Santarem ${ }^{9}$, Anabela Cordeiro-da-Silva ${ }^{9,10}$, Maria Paola Costi ${ }^{6^{*}}$, Alberto Venturelli $i^{3^{*}}$ and Rebecca C. Wade ${ }^{1,2,11,12^{*}}$

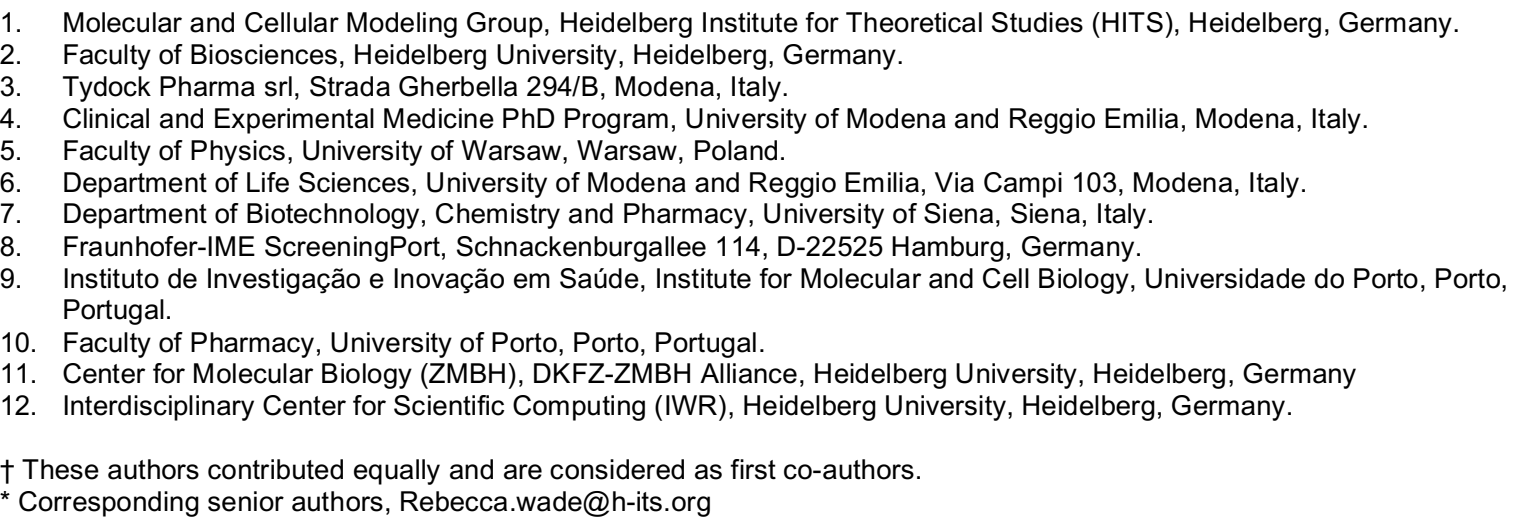

\section{Summary}

The optimization of compounds with multiple targets in the drug discovery cycle is a difficult multidimensional problem. Here, we present a systematic, multidisciplinary approach to the development of selective anti-parasitic compounds. Efficient microwave-assisted synthesis of pteridines along with iterations of crystallographic structure determination were used to validate computational docking predictions and support derivation of a structure-activity relationship for multitarget inhibition. This approach yielded compounds showing picomolar inhibition of $T$. brucei pteridine reductase 1 (PTR1), nanomolar inhibition of L. major PTR1, along with selective submicromolar inhibition of parasitic dihydrofolate reductase (DHFR). Moreover, by combining design for polypharmacology with a property-based on-parasite optimization, we found three compounds that exhibited micromolar $\mathrm{EC}_{50}$ values against $T$. brucei brucei, whilst retaining their target inhibition. Our results provide a basis for the further development of pteridine-based compounds and we expect our multitarget approach to be generally applicable to the design and optimization of anti-infective agents.

\section{Keywords}

Kinetoplastid, antiparasitic inhibitors, folate pathway enzymes, structure-based drug design, virtual screening, microwave assisted organic synthesis, structure-activity relationships, polypharmacology

\section{Abbreviations}

ADMET: Absorption, Distribution, Metabolism, Excretion, Toxicity; DHFR: Dihydrofolate reductase; HAT: Human African Trypanosomiasis; MTX: Methotrexate; NTDs: Neglected Tropical Diseases; PABA: para-amino benzoic acid; PAINS: Pan-Assay Interference compounds; PTR1: Pteridine reductase 1; SI: Selectivity index; TS: Thymidylate synthase 


\section{Introduction}

The World Health Organization has identified 17 Neglected Tropical Diseases (NTDs) that pose a health burden to over 1.4 billion of people. ${ }^{1,2}$ Parasites of the Trypanosomatid family are responsible for two potentially lethal insect-vector borne NTDs: Human African Trypanosomiasis (HAT, sleeping sickness), caused by Trypanosoma brucei, and leishmaniasis, caused by the intracellular parasite Leishmania spp. ${ }^{3-7}$ Current therapeutics are limited by toxicity, poor efficacy and parasite resistance, thus underlining the need for new chemotherapies. ${ }^{8,9}$

One way to identify new anti-parasitic agents is to apply target-based drug design strategies. ${ }^{10-}$ 12 The folate pathway enzyme dihydrofolate reductase (DHFR) is a known anti-cancer, antibacterial and anti-malarial target. ${ }^{13-16}$ It provides reduced folates, which are crucial to biological processes like DNA, protein and amino acid synthesis or one-carbon transfer. ${ }^{14,17,18}$. In Trypanosomatids, DHFR inhibition was found to be ineffective due to a metabolic bypass via the biopterin-reducing pteridine reductase 1 (PTR1, Figure 1): When DHFR is inhibited, PTR1, which can also reduce folates, is overexpressed and sustains sufficient metabolite levels to ensure parasite survival. Thus, when targeting the folate pathway in Leishmania, both DHFR and PTR1 need to be considered. ${ }^{19-21}$ In T. brucei, PTR1 was shown to be a potential antiparasitic target in its own right by RNA interference studies. ${ }^{22,23}$ Nonetheless, even nanomolar PTR1 inhibitors have so far shown limited anti-parasitic activity in vitro ${ }^{24,25}$, suggesting that targeting the $T$. brucei folate pathway may also benefit from the consideration of both PTR1 and DHFR.

Screening a set of folate-related compounds against parasitic folate pathway targets previously led to the identification of compounds $\mathbf{6 a}$ (methyl-1-(4-(((2,4-diaminopteridin-6$\mathrm{yl}$ )methyl)amino)benzoyl)piperidine-4-carboxylate, herein compound 2) and 6b (methyl-1-(4(((2,4-diaminopteridin-6-yl) methyl) (methyl) amino) benzoyl) piperidine-4-carboxylate, herein compound 1) as submicromolar inhibitors of Leishmania major PTR1 (LmPTR1) with $\mathrm{K}_{i}$ values of $0.10 \mu \mathrm{M}$ and $0.04 \mu \mathrm{M}$, respectively. ${ }^{26} 2$ was additionally a micromolar inhibitor of $L$. major DHFR (LmDHFR) with a weak selectivity for the parasite enzyme over the human DHFR (hDHFR) ( $\mathrm{K}_{\mathrm{i}}$ of $4 \mu \mathrm{M}$ vs. $10 \mu \mathrm{M}$ ). In contrast to the parasite DHFR, which is covalently coupled with thymidylate synthase (TS) in a bifunctional DHFR-TS, the hDHFR off-target is monofunctional and shares only about $30 \%$ sequence identity with parasite DHFR domains, indicating potential for further selectivity optimization. ${ }^{27-29}$

The aim of the current work was to optimize pteridine-based compounds for their inhibition of T. brucei PTR1 (TbPTR1) and TbDHFR, in addition to the Leishmania targets, while ensuring selectivity against the off-target hDHFR. The enzymatic evaluation of reference pteridines reported earlier ${ }^{26,30}$ and our published comparative study of trypanosomatid folate pathway proteins $^{31}$ supported the design of three series of compounds to explore substituents at three 
positions on the pteridine structure, and a fourth 'merged' series containing permutations of the substituents in the three series. Docking simulations and three new crystallographic complexes of pteridines with TbPTR1 and a complex with LmPTR1, supported the targetbased design approach and the determination of structure-activity relationships. A systematic analysis of correlations between computed physicochemical molecular descriptors and observed anti-parasitic effects allowed us to prioritize promising compounds for synthesis. In total, 26 new pteridine derivatives were characterized experimentally, most of which showed improved target inhibitory profiles for PTR1 and DHFR of both L. major and T. brucei. Among these, we report the first, to the best of our knowledge, picomolar inhibitors of TbPTR1 and several new low nanomolar inhibitors of LmPTR1, which mostly also show selective micromolar to submicromolar inhibition of the parasite DHFR variants. In vitro evaluations of the effect on $T$. brucei brucei bloodstream forms revealed three new inhibitors with low micromolar to submicromolar $\mathrm{EC}_{50}$ values against the $T$. brucei parasite.

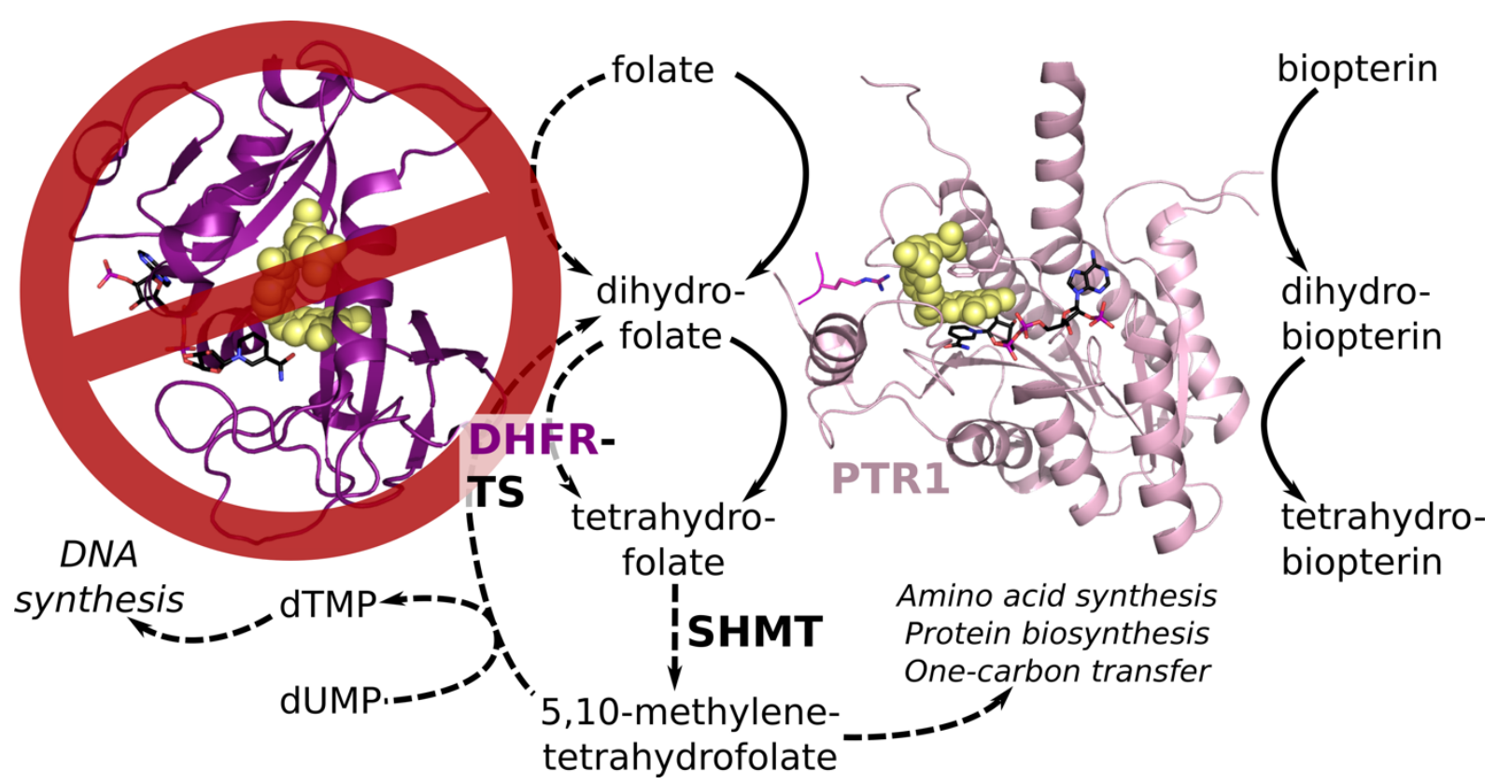

Figure 1. Overview of the pterin activation in the Trypanosomatidic folate pathway when DHFR is inhibited and PTR1 provides a metabolic bypass. The DHFR domain of the bifunctional DHFR-TS would, under normal conditions, reduce biological folates to tetrahydrofolate (THF). THF is converted to 5,10-methylene THF by the serine hydroxymethyl transferase (SHMT) and this metabolite has a central role in amino acid synthesis, protein biosynthesis and one-carbon transfer. It is also required by the TS domain of DHFR-TS to convert deoxyuridine monophosphate (dUMP) to deoxythymidine monophosphate (dTMP), which is necessary for DNA synthesis. PTR1, which primarily reduces unconjugated pterins like biopterin, takes over folate reduction when DHFR is inhibited, thus acting as a metabolic bypass and an important additional target that needs to be inhibited for shutting down the Trypanosomatidic folate pathway. Both proteins are shown in cartoon representation (DHFR domain of DHFR-TS: purple, PTR1: light pink, single monomer of functional tetramer) with the NADPH/NADP ${ }^{+}$ cofactor in sticks with black carbons and the folate substrate in yellow spheres. In PTR1, in addition, an arginine residue from a neighboring subunit pointing into the active site is shown in magenta sticks. 


\section{Results}

\section{Reference compounds inhibit both PTR1 and DHFR and adopt a methotrexate-like}

\section{binding mode}

To systematically assess multitarget inhibition, we measured the inhibition of TbPTR1, TbDHFR, LmPTR1, LmDHFR, and the off-targets hDHFR and hTS, by the folate-related anticancer agent methotrexate (MTX) and 7 pteridine-based reference compounds (1, 2, III-VII, Figure $\mathbf{2 A B}$ and Table $\mathbf{S 1}, \mathrm{SI}){ }^{26,30,32}$ Although the seven reference compounds were primarily

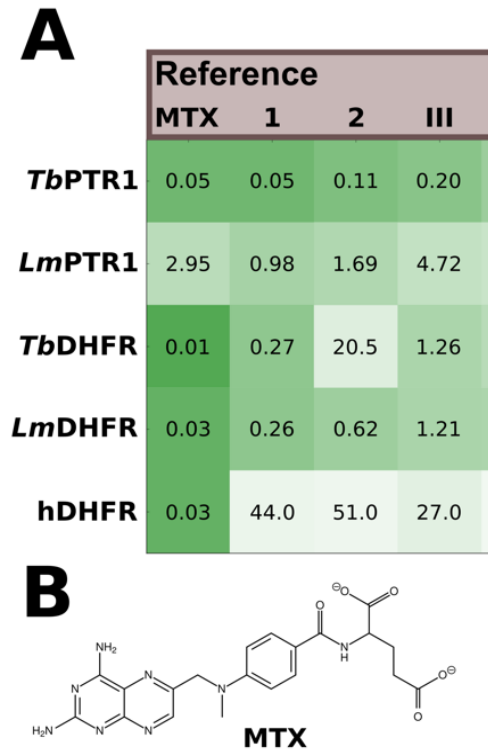

MTX<smiles>CCC(=O)C1CCC(C(=O)c2ccc(CC(C)c3ccc4cc(C)nc(C)c4n3)cc2)CC1</smiles>

1<smiles>[R]c1ccc(C(=O)C2CCC(C(=O)OC)CC2)cc1</smiles>

2<smiles>C#CCCc1ccc(CC(=O)c2ccc3cc(C)nc(O)c3c2)cc1</smiles><smiles>CC(C)=CC(C)C</smiles>

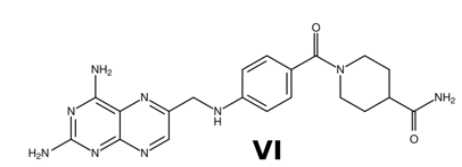<smiles>CCOC(=O)C1CCCC1C(=O)c1ccc(CNc2ccc3cc(C)cc(O)c3n2)cc1</smiles>
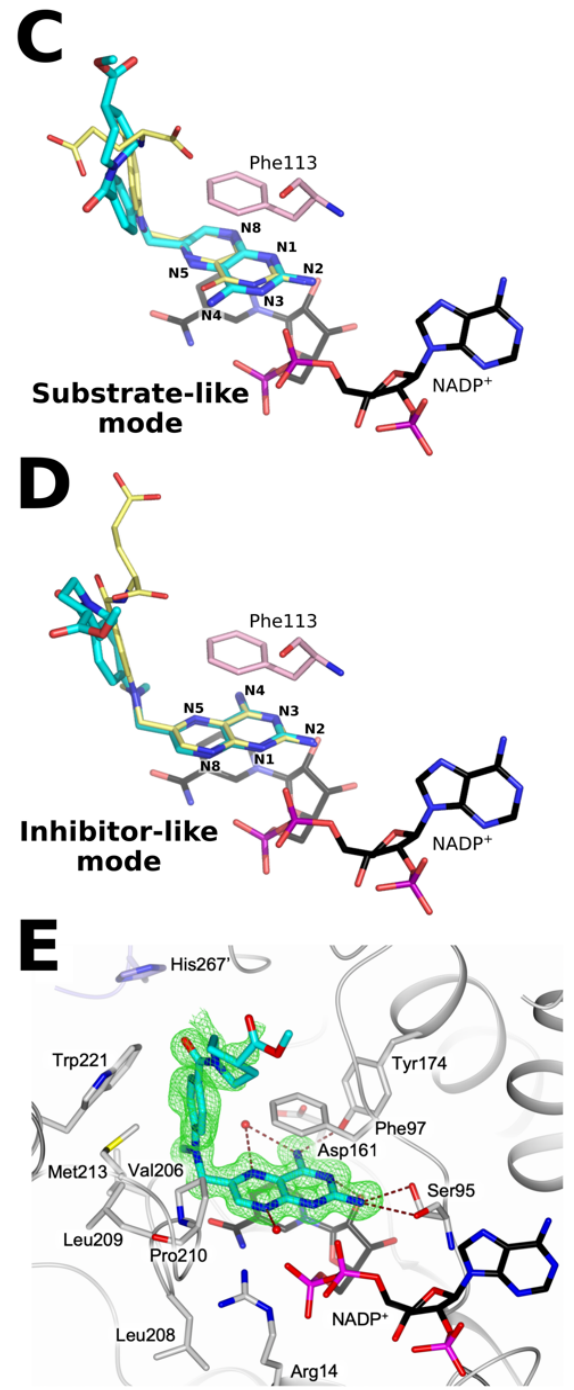

Figure 2. Inhibitory activities, structures and orientations of reference pteridines in crystal structures of $L m P T R 1$ (PDB-ID 2qhx) and TbPTR1 (PDB-ID 6rx5). (A) The activity heatmap shows IC 50 values in different targets and offtargets in $\mu \mathrm{M}$, colored from green to white for low to high IC ${ }_{50}$ s. Values are reported for TbPTR1, LmPTR1, TbDHFR, LmDHFR and the off-target hDHFR. All values, as well as data for hTS, are reported in Table S1, SI. (B) The previously published compounds shown were used as references: 1, 2 and VII are 6 b, 6a and 6c from Cavazzuti et al. ${ }^{26}$ and III, IV, V and VI correspond to $\mathbf{5 d}, \mathbf{5 b}, \mathbf{6 a}$ and $\mathbf{5 a}$ from Corona et al. ${ }^{30}$ (C) Compound $\mathbf{1}$ (cyan carbons) in its substrate-like conformation in LmPTR1 with folate superimposed from TbPTR1 PDB-ID 3bmc (yellow sticks); (D) Compound 1 (cyan carbons) in an inhibitor- or MTX-like orientation in LmPTR1 with MTX superimposed from LmPTR1 PDB-ID 1e7w (yellow sticks). The pteridine nitrogens are labeled according to the ring nomenclature. (E) Crystallographic complex of TbPTR1 (gray cartoon, His267' from the neighboring subunit in lavender) in complex with NADPH/NADP ${ }^{+}$and compound 1 determined in this work. In TbPTR1, compound 1 shows the MTX-like binding mode. Interacting residues (in C, D: only Phe113) and the NADPH/NADP ${ }^{+}$cofactor are shown in sticks (carbons colored according to protein and black, respectively). In (E), water molecules are shown as red spheres and the inhibitor is surrounded by the omit map (green wire) contoured at the $2.5 \sigma$ level. Hydrogen bonds are represented by brown dashed lines. 
designed as LmPTR1 inhibitors, we found all except VII to be more potent against TbPTR1 than LmPTR1. 1 was the strongest inhibitor of TbPTR1 with an $\mathrm{IC}_{50}$ of $50 \mathrm{nM}$ against TbPTR1 and $1 \mu \mathrm{M}$ against $L m$ PTR1 (Figure 2A).

Notably, the reference compounds largely exhibited micromolar to submicromolar inhibition of LmDHFR and TbDHFR (IC 50 LmDHFR 0.3 - $1.4 \mu \mathrm{M}$; TbDHFR $0.3-20.5 \mu \mathrm{M}$ ). While MTX was more potent against the parasite DHFRs, it was not selective (Selectivity index SI: TbDHFR/hDHFR 3 and $L m D H F R / h D H F R ~ 1)$. Among the reference compounds, we observed selectivity indices of up to about 160-170 for TbDHFR and $L m D H F R$.

Previously determined crystal structures show that compounds $\mathbf{1}$ and $\mathbf{2}$ share a substrate-like pterin orientation in the complex with LmPTR $1 .{ }^{26}$ Compound 1, in addition, features a second, so-called inhibitor-like (or MTX-like) orientation, with the bicyclic ring system flipped by $180^{\circ}$ and rotated by $30^{\circ}$ (Figures $2 \mathrm{CD}$ and S1 of the SI). ${ }^{26}$ Similar observations have been made in crystallographic complexes of TbPTR1 with small pteridine-based inhibitors. ${ }^{32}$ Our crystal structure of the ternary complex of TbPTR1 with NADPH/NADP ${ }^{+}$and the reference compound 1 (PDB-ID 6rx5, resolution $1.42 \AA$, experimental details: Tables S2-S3, SI) confirms that the diaminopteridinyl moiety of 1 adopts only the MTX-like orientation (Figure 2E), resembling its MTX-like binding mode in LmPTR1 (Figures 2D and S2, SI). Docking studies consistently indicated that the reference compounds adopt MTX-like binding modes in the different targets and the off-target hDHFR (Table S4, Figure S5, SI). Since the reference compounds showed micromolar to submicromolar inhibition of parasitic PTR1 and DHFR, whilst being modestly selective for parasite over hDHFR, we next aimed to improve the target inhibition profiles in a multitarget-based design approach.

\section{Four series of compounds designed to improve dual target selective inhibition}

To optimize the compounds for TbPTR1/TbDHFR and LmPTR1/LmDHFR while minimizing inhibition of hDHFR, we employed our published optimization guidelines for MTX-like scaffolds derived from the extensive comparison of on- and off-targets in the parasitic folate pathway: ${ }^{31}$ Based on the overlapping properties in the different protein targets and differences between targets and off-targets, modifications for improved target interactions and selectivity were defined for 1 (Figure 3A). We designed three series of compounds, each with modifications in one of three parts of the pteridines: the N10 position, the para-amino benzoic acid (PABA) moiety and the tail portion (Figure 3B).

In the N10 series, five novel pteridines (3-5, 9 and 22, Figure 3B) were modified in the N10 position to improve interactions with PTR1 and parasite DHFR and to exploit the differing pocket sizes and residues in parasitic targets and the hDHFR off-target. ${ }^{31}$ Nonpolar substitutions, like the ethyl and propargyl substituents of $\mathbf{3}$ and $\mathbf{5}$ were designed to interact with aliphatic residues surrounding N10 in the parasite targets, e.g. Leu209 of TbPTR1; Ile47 and 


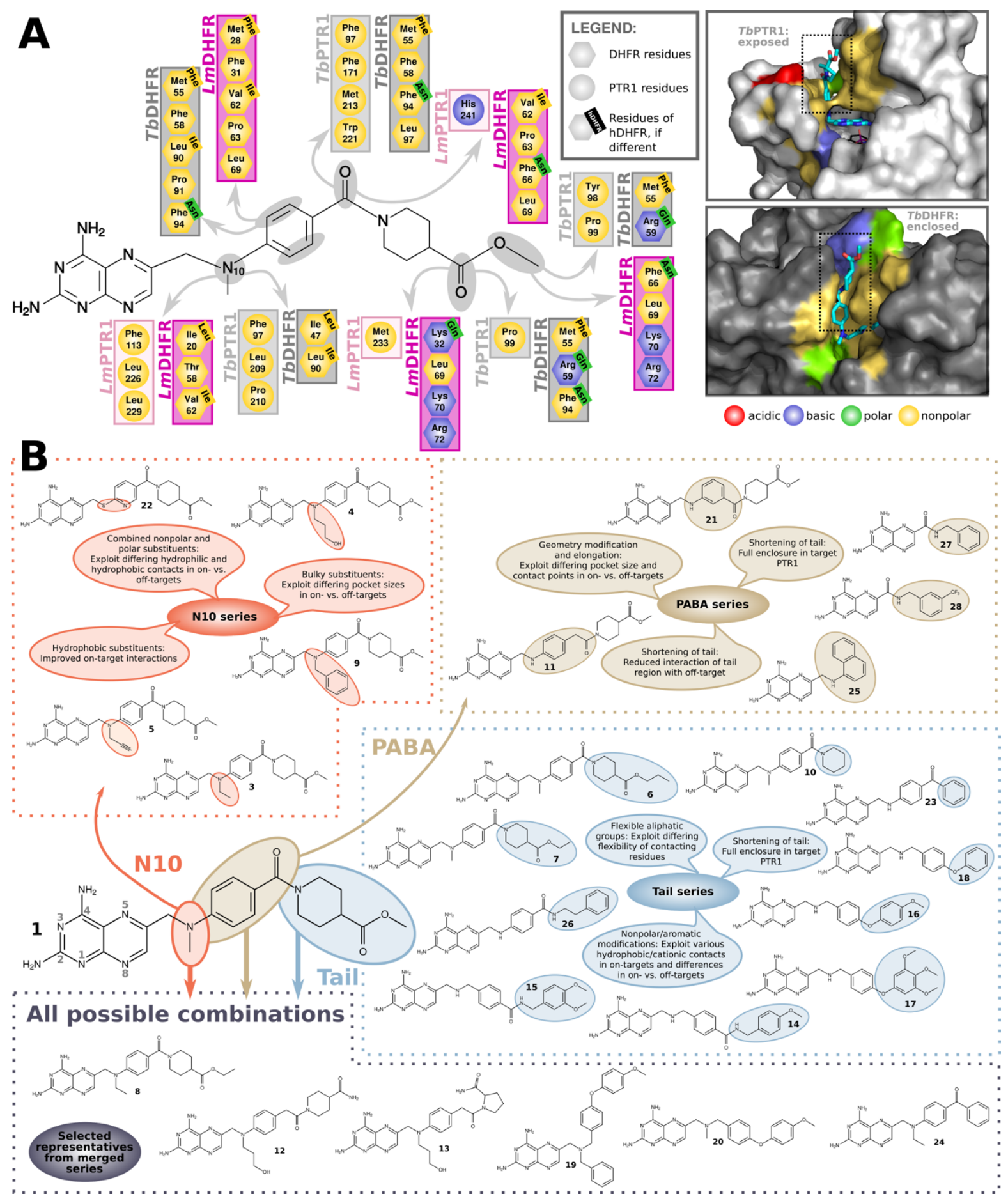

Figure 3. Structural features of PTR1 and DHFR considered in the multitarget design of selective compounds and overview of the modifications explored in four designed compound series, exemplified by highlighting on reference compound 1. (A) Selected residues within $5 \AA$ of the marked modification sites based on the bound orientations of 1 in TbPTR1 (pale gray), TbDHFR (dark gray), LmPTR1 (light pink) and LmDHFR (magenta). Based on Panecka-Hofman et al. ${ }^{31}$, this map provides an overview of residues having similar properties in the surrounding of specific ligand portions in all targets (covering only those applied for the design as shown in (B), for full maps, see Figures S3,S4). In some positions, parasite DHFR differs in amino acid type from the offtarget hDHFR, thus highlighting suitable substitution points to improve selectivity. Differing hDHFR residues are labeled in the top right corner of the corresponding parasite DHFR residue. A surface representation of complexes of 1 with TbPTR1 (top right, PDB-ID 6rx5) and TbDHFR (bottom right, MTX-like docking result that was top-ranked by the Glide docking score in PDB-ID 3rg9) highlights the solvent-exposure of the tail in PTR1, which is in contrast well-enclosed in DHFR. All residues in (A) are colored by type: red=acidic, blue=basic, green=polar, yellow=nonpolar. Compound 1 and the NADPH/NADP ${ }^{+}$cofactor are shown in sticks with cyan and black carbons, respectively. (B) Synthesized members of each designed series with the series for the three modification sites (N10, PABA, tail) shown in a framed box along with the key objectives addressed with the respective modifications. A fourth, merged series, shown at the bottom of the figure, was constructed by combining all modifications studied in silico in all possible permutations. Of the resulting 2014 compounds, six selected representatives were synthesized. The small grav numbers indicate the pteridine ring nomenclature. 
The large benzyl substituent of 9 may in addition allow for enhanced selectivity due to the lower volume of the hDHFR compared to the parasite DHFR pocket (pocket volume TbDHFR 353 $\AA^{3}$, LmDHFR $384 \AA^{3}$ and hDHFR $347 \AA^{3}$ ). In 22, we replaced N10 by sulfur and the PABA benzene ring by pyridine, to improve off-target selectivity. Whereas hDHFR favors hydrogen bond donors in the proximity of N10 and the PABA ring system, the parasite DHFRs show regions favorable for interaction with hydrogen bond acceptors. ${ }^{31}$ Corona et al. ${ }^{30}$ demonstrated improved selectivity for PTR1 over hDHFR by hydrophilic N10 substitutions. Our data for reference compound III, with a hydroxyethyl substituent, did not confirm this conclusion (Figure 2A) and our docking simulations indicated that interaction with a conserved structural water might require an unfavorable conformation of the substituent (Figure S5A, SI). To improve interactions between the substituent and water, we therefore elongated the aliphatic linkage to a hydroxypropyl in 4.

In the PABA series with 5 new pteridine derivatives $(11,21,25,27-28$, Figure 3B), we explored modifications of the PABA moiety and the amide linkage to the tail portion. To improve selectivity by exploiting the different pocket sizes and contact points of hDHFR and the parasite proteins, in 11, we replaced the PABA phenyl group with benzyl and, in 21, the entire PABA moiety by a meta-aminobenzoic acid (Figure 3 ). The compound tails are solvent-exposed in PTR1, thus having poorly defined interactions (Figure 3A), whereas strong interactions occur with the hDHFR off-target. ${ }^{31}$ We therefore shortened the tail region to achieve full enclosure in the PTR1 binding pocket by replacing PABA by naphthalene or benzene (non-substituted or substituted with $-\mathrm{CF}_{3} ; 25,27$ and 28 , respectively).

In the tail series, we explored tail modifications resulting in 10 additional new pteridines (6-7, 10, 14-18, 23 and 26, Figure 3B). In both $T$. brucei targets, hydrophobic contact points accessible to the compound tail region exist (Figure $\mathbf{3 A}$ ). Since the flexibility of the tail likely contributes entropically to binding affinity in PTR1, directional interactions of the tail may be unfavorable. Hydrophobic interactions are geometrically less restrained than, for instance, hydrogen bonds, and thus less likely to entropically penalize binding. Therefore, the methyl ester in the tail of $\mathbf{1}$ was replaced by the more flexible ethyl and propyl in $\mathbf{7}$ and $\mathbf{6}$, respectively. Additionally, in parasite DHFR, the tail is enclosed by more hydrophobic moieties than in the hDHFR off-target (Figure 3A) and the surrounding residues show different conformational variability in the crystal structures, which suggests further on/off-target selectivity benefits. ${ }^{31}$ Combining the exploitation of these differences with improved enclosure in PTR1 (Figure 3A), we modified the tail to an unsubstituted piperidine (10) or replaced piperidine with an unsubstituted benzene (23). Compound 26, with benzene attached via a flexible ethyl linkage to an MTX-like amide, can benefit from nonpolar and aromatic contact points in PTR1 and DHFR and adapt to their differing placement. The flexible aromatic tail may further form cation$\pi$ interactions with positively charged residues in the entrance of the DHFR pocket (Figure 
3A). In compounds 14 and 15, we explored an altered geometry with a one-carbon spacer between N10 and PABA and amide-linked methoxylated tail portions (Figure 3B). The methoxylations may form additional contacts with hydrophobic residues in the target pocket entrance regions (e.g. Pro99 of TbPTR1, Figure 3A). In addition, an etheryl linkage to a nonsubstituted (18), methoxylated (16) or trimethoxylated (17) benzyl group was explored to likewise exploit hydrophobic, aromatic and positively charged contact points found around the tail region in the various targets (Figure $3 \mathbf{A}$ ).

In a fourth series, the 'merged' series, the modifications in the above three series were permuted and merged by decomposing the compounds into fragments and recombining them in silico in all possible combinations of these fragments (see details in the SI). The 2014 compounds generated were filtered based on docking results and physico-chemical properties and, of the remaining 600 candidates, six were selected for synthesis: Two compounds (12 and 13, Figure 3B) were chosen for their favorable interaction patterns and scores predicted by docking simulations, while four other compounds (8, 19, 20 and 24, Figure 3B) were selected based on physicochemical marker properties that show some correlation with in vitro anti-parasitic activity as described below. 12 combined the N10 hydroxypropyl substituent of $\mathbf{4}$, the tail carboxamide group of $\mathbf{V I}$ and the elongation by a carbon spacer in the PABA moiety of 11. The same combination with a pyrrolidine replacing piperidine, like in reference compound VII, additionally gave 13. Similarly, the ethyl modification of N10 in $\mathbf{3}$ and the ethyl ester in the tail fragment of $\mathbf{7}$ were joined to give $\mathbf{8}$, which largely addressed the hydrophobic contact points in different targets. The ethyl modification of $\mathrm{N} 10$ was also joined with the shortened tail of $\mathbf{2 3}$ to give compound $\mathbf{2 4}$. Finally, the N10 benzyl-substitution of $\mathbf{9}$ and the methyl of the reference compound 1 were joined with the PABA and tail-modified scaffold of 16 to give 19 and 20 , respectively.

\section{Synthesis of pteridine derivatives with high yield}

The synthesis of the 26 new 2,4-diaminopteridine derivatives and resynthesis of the reference compounds 1 and 2 is reported in detail in the SI, including synthetic schemes S1-S8. We applied our microwave-assisted methodology for improving the reaction yield of the chemical pteroid step to obtain compounds 1-26 in high yields of $70-90 \%$ with reduced reaction time. ${ }^{33}$ For most compounds, the PABA amine functionalization was achieved via selective alkylation using nitriles as an alkylating reagent with $\mathrm{Pd} / \mathrm{C}$ or conventional alkylation with propargyl bromide or (bromomethyl) benzene. ${ }^{34,35}$ Fischer esterification was employed for the isonipecotic acid derivatization in the tail moiety. 


\section{Designed pteridine derivatives have improved on-target and off-target enzyme inhibitory activity profiles and bind in an MTX-like orientation}

The measured inhibitory activities of compounds 3-28 against the targets TbPTR1, TbDHFR, LmPTR1, LmDHFR, and the off-targets hDHFR and hTS, are given in Figure 4 and Table S1. Most of the new pteridine derivatives displayed 1-2-fold greater inhibition of TbPTR1 than $L m P T R 1$ and were more or equally active against PTR1 than the reference compound 1 . The inhibitors with nanomolar to picomolar PTR1 inhibition showed improved selectivity for PTR1 over the off-target hDHFR by up to about 3 orders of magnitude. The activities against TbDHFR and $L m D H F R$ exceeded those against hDHFR, where the $I_{50}$ was typically worse than 100 $\mu \mathrm{M}$. For parasite DHFR, the best compounds were overall similar to 1: compound 8 with an $I_{50}$ of $0.23 \mu \mathrm{M}$ against TbDHFR and 6, 7, 8 and 24 with $I_{50}$ S of $0.13-0.23 \mu \mathrm{M}$ against LmDHFR (compared to 1: $0.3 \mu \mathrm{M}$ for both TbDHFR and LmDHFR). Only compound 13 was slightly more selective for TbDHFR than 1 with an SI of 169 (compared to 164). Docking studies suggested that the majority of the novel pteridines adopted MTX-like binding modes in both PTR1 and parasite DHFR variants and the off-target hDHFR (see docking results, Tables S5, S6, SI).

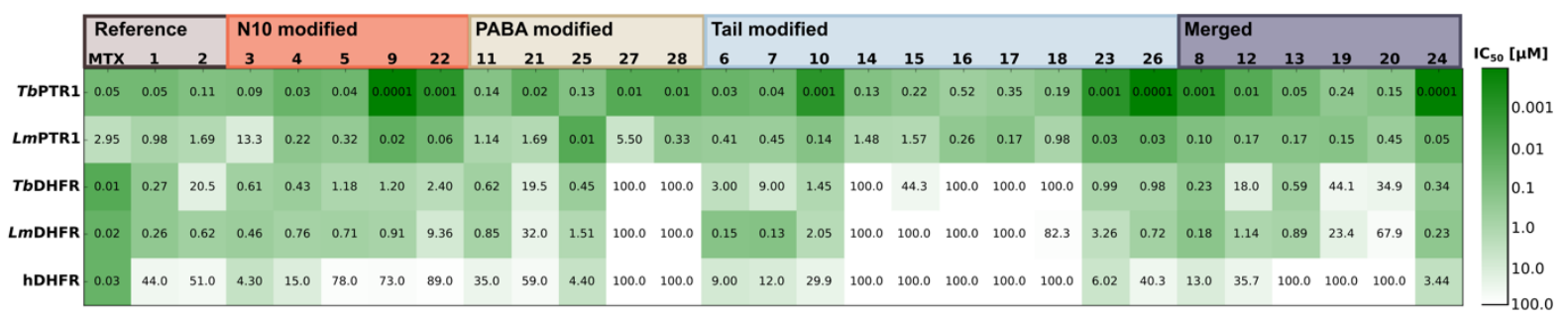

Figure 4. Inhibitory activities of compounds of the designed N10-, PABA-, and tail-modified series, the merged series, and selected reference compounds. The activity heatmap shows $\mathrm{IC}_{50}$ values in different targets and offtargets in $\mu \mathrm{M}$, colored from green to white for low to high $\mathrm{IC}_{50}$ s. Values are reported for TbPTR1, LmPTR1, TbDHFR, LmDHFR and the off-target hDHFR. All values, as well as data for hTS, are reported in Table S1.

Pteridine derivatives 3 and 4 bind in an MTX-like orientation in both PTR1 variants. The structures of TbPTR1 with the new pteridines $\mathbf{3}$ and $\mathbf{4}$, and that of LmPTR1 with $\mathbf{4}$, were determined to $1.20 \AA, 1.11 \AA$ and $2.10 \AA$ resolution, respectively (see Tables S2-S3). The overall structure of the TbPTR1 complexes resembles the complex with 1 (compare Figure 2E with $\mathbf{5 A}$ ). The $\mathrm{N}$-ethyl moiety of $\mathbf{3}$ forms van der Waals interactions with Val206 and Trp221 on the hydrophobic side of the pocket (Figure 5A). The bulkier N-propylhydroxyl moiety of 4 forms direct and water-mediated hydrogen bonds with Asp161 and receives an intramolecular hydrogen bond from the amine in position 4 on the pteridine system.

The structural characterization of LmPTR1 in complex with 4 (Figure 5B) shows the presence of a functional enzyme tetramer in the crystal asymmetric unit with a similar structure to those previously determined. ${ }^{36,37}$ The MTX-like binding mode adopted by $\mathbf{4}$ in LmPTR1 closely resembles that observed in TbPTR1 except for the terminal piperidine moiety (Figure S2CD of 
the SI). The latter is highly flexible and was only poorly visible in our models - a possible orientation is reported, but further orientations cannot be excluded.

N10 modifications yield improved PTR1 inhibitors with similar trends for DHFR selectivity. The N10-modified compounds (3-5, 9 and 22; Figure 3B) were improved PTR1 inhibitors in comparison to 1 with the exception of 3 (1 IC 50 TbPTR1 $50 \mathrm{nM}$, LmPTR1 $1 \mu \mathrm{M}$; N10 series $\mathrm{IC}_{50}$ TbPTR1 $<0.1$ - $90 \mathrm{nM}$; LmPTR1 0.02-13.3 $\left.\mu \mathrm{M}\right) .9$ was the best in the series with a picomolar $I_{50}$ against TbPTR1 and an $I_{50}$ of $20 \mathrm{nM}$ against $L m P T R 1$. The predicted orientations of the compounds in PTR1 were overall similar to the crystallographic data (Figure 5AB), commonly showing the MTX-like orientation. 9, when docked in presence of conserved structural water, was an exception due to its bulky benzyl substituent and required the reorganization of Trp221 in TbPTR1 (see the induced fit docking pose in Figure 5C). This reorganization is plausible since Trp221 was previously identified as a flexible residue in the TbPTR1 pocket gating region on the basis of crystallographic data. ${ }^{31}$

All compounds were roughly similar to 1 in parasite DHFR inhibition ( 1 IC 50 TbDHFR and LmDHFR $0.3 \mu \mathrm{M}$; N10 series $\mathrm{IC}_{50}$ TbDHFR 0.4-2.4 $\mu \mathrm{M}$, LmDHFR 0.5-9.4 $\mu \mathrm{M}$ ). The trends observed for parasite DHFR were however reflected in similar changes of the hDHFR inhibition and selectivity ranged from 7- to 66-fold for TbDHFR and 9- to 110-fold for LmDHFR over hDHFR, which was lower than for 1 (SI TbDHFR/hDHFR 164 and LmDHFR/hDHFR 167). Thus, mainly PTR1 inhibition benefited from the selected N10 modifications.

\section{PABA modifications lead to strong variations in the target inhibition profile.}

The modifications of the PABA moiety in the PABA series (compounds 11, 21, 25, 27 and 28; Figure 3B) distinctly affected the target activities. Particularly smaller, well-enclosed compounds showed varying activity improvement for different PTR1s: 25 , in contrast to most of the studied pteridines, was 13-fold more potent towards LmPTR1 than TbPTR1 (IC $5010 \mathrm{nM}$ and $130 \mathrm{nM}$, respectively) and benefits from its steric fit to the elongated LmPTR1 binding pocket (Figure 5DF). The small, fully enclosed 27 and 28 were stabilized by hydrophobic residues lining the pocket entrance of TbPTR1 (see Figure 3A), which results in an around 10fold higher potency than for reference compound 2 with the corresponding N10 substitution (IC50 27, 28: $10 \mathrm{nM}$; 2: $110 \mathrm{nM})$. 


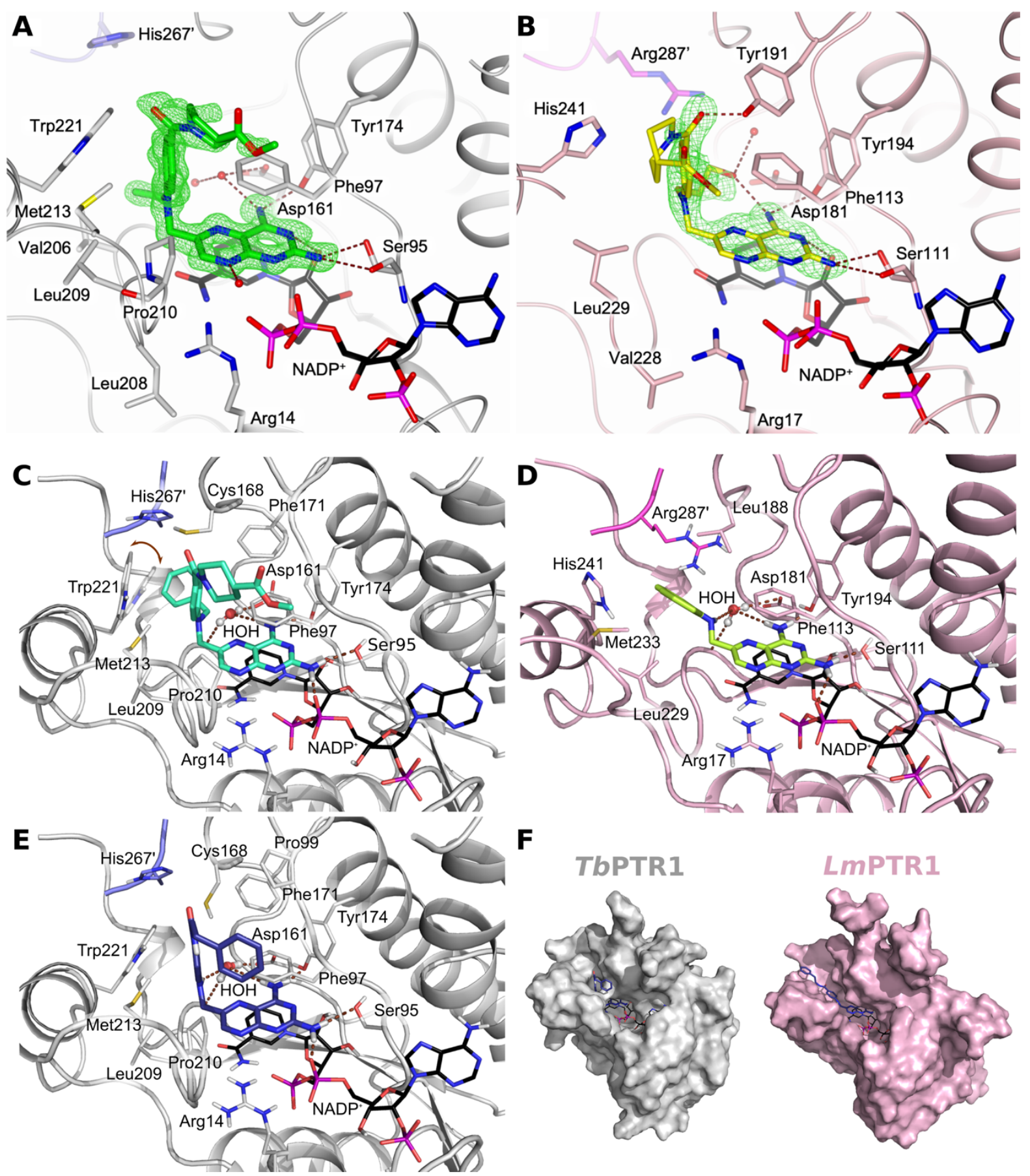

Figure 5. Orientations of pteridine-based inhibitors in TbPTR1 and LmPTR1 based on crystallographic complexes (A, B) and docking solutions (C-E) and comparison of pocket shape for the PTR1 variants (F). (A, B) Crystallographic complexes determined in this work show the MTX-like binding modes for both TbPTR1 (A, gray cartoon, His267' from the neighboring subunit in lavender) and LmPTR1 (B, pink cartoon, Arg287' from the neighboring subunit in magenta) in complex with NADPH/NADP ${ }^{+}$and the inhibitors 3 ( $A$, green) and 4 (B, yellow). Water molecules are shown as red spheres and the inhibitors are surrounded by the omit map (green wire) contoured at the $2.5 \sigma$ level. (C) Induced fit (IF) MTX-like docking solution for compound 9 in TbPTR1 in the presence of a conserved water molecule (ball-and-stick representation): Trp221 moves (indicated by a brown arrow) to make room for the phenyl of 9. (D, E) Docking poses of: 25 (D, lime carbons) in $L m P T R 1$ and 26 (E, dark purple carbons) in TbPTR1. All presented docking solutions were obtained in the presence of conserved structural water. Similar poses were generally observed in the docking without water. (F) Differing shape of the binding pocket in the two PTR1 variants: The compounds (in the example, 26) show conserved core interactions in both PTR1s and their tails orient along the elongated binding site funnel in $L m P T R 1$, whereas the tail conformations in TbPTR1 are typically kinked. Proteins are shown in surface representation. In all panels, interacting residues and the NADPH/NADP+ cofactor are shown in sticks (carbons colored according to protein and black, respectively). Hydrogen bonds are represented by brown dashed lines. Docking results are only presented for N1-deprotonated compounds, but similar orientations were observed for the N1protonated variants (see Fiqure S6). Further IF docking solutions are shown in Fiqures S7, S8. 
Against $L m D H F R, 25$ showed similar activity to 2 ( I $_{50} 1.5$ and $0.6 \mu \mathrm{M}$, respectively), whereas it displayed higher activities for both TbDHFR (IC 50 2: $20.5 \mu \mathrm{M} ; 25: 0.5 \mu \mathrm{M})$ and hDHFR $\left(\mathrm{IC}_{50}\right.$ 2: $51 \mu \mathrm{M} ; 25: 4 \mu \mathrm{M}$ ). A one-carbon spacer to shift the position of the PABA carbonyl in 11 retained the $L m D H F R$ activity similar to $2\left(11: I_{50} 0.8 \mu \mathrm{M}\right)$ but increased the inhibition of TbDHFR ( $\left.\mathrm{IC}_{50} 0.6 \mu \mathrm{M}\right)$. While the compounds were generally well stabilized by hydrophobic interactions in all DHFR variants, differences in pocket size and surrounding residue patterns in the proximity of the PABA moiety (Figure $3 \mathrm{~A}$ ) modulate the differing activity profiles.

\section{Alterations in tail geometry boost PTR1 inhibition but can reduce DHFR activity.}

In the tail-modified series (compounds 6-7, 10, 14-18, 23 and 26; Figure 3B), hydrophobic and aromatic residues lining the pocket entrance region of PTR1, like Tyr98, Pro99 and Phe171 of TbPTR1 or Met233 and His241 of LmPTR1 (Figure 3A) were exploited by either tail elongation or shortening. Interactions of these residues with the flexible aromatic tail of 26 (Figure 5EF) likely contribute to the boost of the $\mathrm{IC}_{50}$ against TbPTR1 to the picomolar range and to $30 \mathrm{nM}$ against $L m P T R 1$ - a more than 1000-fold and 57-fold improvement, respectively, in PTR1inhibitory potencies compared to reference compound 2 . The shortened tails of 10 (unsubstituted piperidine) and $\mathbf{2 3}$ (benzene) were stabilized by the same residues and benefit from a better enclosure in the PTR1 pocket. Both compounds show increased TbPTR1 and LmPTR1 inhibition when compared to 1 (IC ${ }_{50}$ TbPTR1 10, 23: 1 nM both vs. 1: $50 \mathrm{nM}$; LmPTR1 10: $0.1 \mu \mathrm{M}, 23$ : $0.03 \mu \mathrm{M}$ vs. 1: $1.0 \mu \mathrm{M})$.

Tail shortening diminished the inhibition of parasite DHFR, whereas it did not affect or even increased the effect on the off-target hDHFR. The piperidine/benzene groups in the tails of 10 and 23 can form more extended hydrophobic interactions with Phe31 of hDHFR than with the corresponding methionine in the parasite DHFR variants (Figure 6). In the parasite protein, moreover, Asn64 in the pocket entrance of hDHFR is replaced by phenylalanine, which, upon interaction with the compound tail, becomes solvent-exposed. The aforementioned pocket size and interaction pattern differences between $L m$ DHFR and other DHFR variants (Figure 3A) also affected the tail series: For instance, $\mathbf{2 3}$ was more active against both TbDHFR and hDHFR than 2 ( $\mathrm{IC}_{50}$ TbDHFR 1 vs. $21 \mu \mathrm{M}$, hDHFR 6 vs. $\left.51 \mu \mathrm{M}\right)$, while both compounds showed similar activity for $L m D H F R$.

\section{Specific combinations of modifications lead to improved on-target inhibition and selectivity.}

Since the crystal structures and docking studies suggested a preference of the MTX-like orientation in all receptors, we used constrained docking to evaluate this binding mode for all 2014 members of the in silico library, containing all possible combinations of merged compound fragments explored in the N10, PABA and tail series. In most cases, MTX-like 
orientations with good scores were obtained and, therefore, the docking alone did not allow us to rule out many of the compounds. However, by combining a docking criterion with property and liability filters, we obtained 600 promising candidates composed of fragments of existing compounds in new combinations (Figure 7A). Of these, six compounds with a diverse set of properties were selected for synthesis and further studies based on expert opinion.

Compounds 12 and 13 (Figure 3B) combined fragments of 4, 11, reference VI, and, for 13, in addition, reference VII. The activities and predicted interactions in all parasite targets were most reminiscent of $\mathbf{4}$, suggesting the key importance of the hydroxy-propyl substituent to N10 for the target inhibition. Notably, while 12 was poorly selective for TbDHFR (2-fold) and modestly selective for $L m D H F R$ (31-fold), 13 was inactive against hDHFR, resulting in a SI of 170 and 113 for TbDHFR and LmDHFR, respectively. Moreover, the compound had Sls over hDHFR of about 2000 for TbPTR1 and 588 for LmPTR1. However, in contrast to most compounds, 13 displayed a weak inhibition of hTS $\left(\mathrm{IC}_{50} 29 \mu \mathrm{M}\right)$.
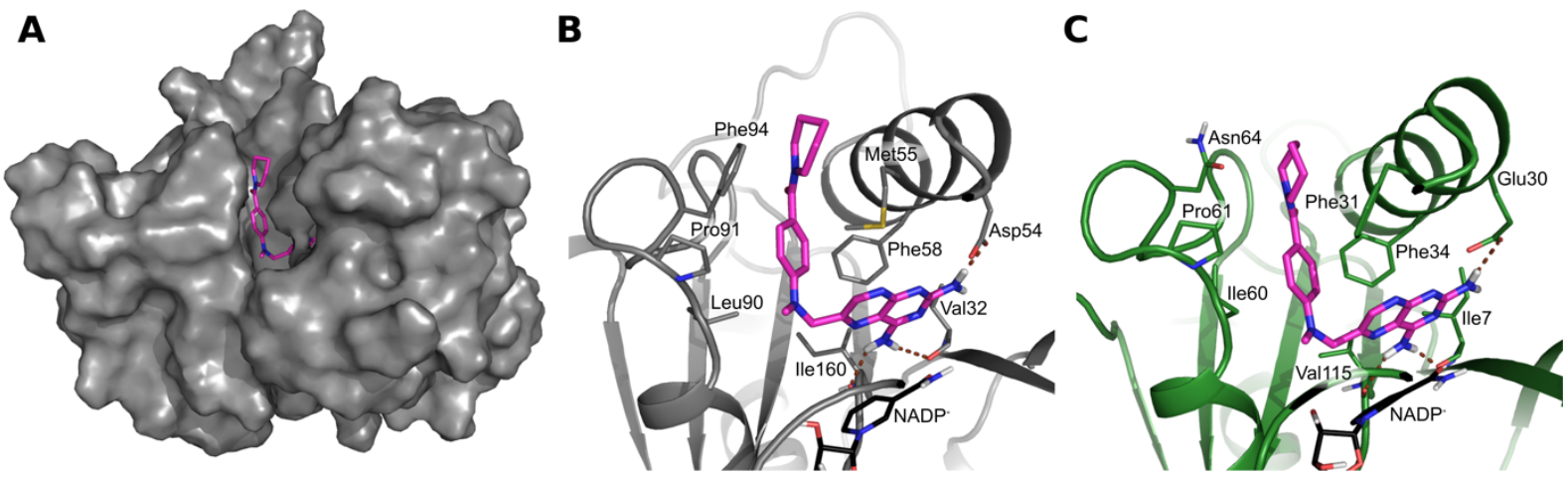

Figure 6. Docking results for compound 10 from the tail series (magenta sticks) in TbDHFR (A, B) and hDHFR (C). (A) The TbDHFR pocket accommodates 10 with its tail enclosed by surrounding residues. hDHFR has a similar shape. TbDHFR is shown in gray surface representation. (B, C) TbDHFR and hDHFR are shown in cartoon representation in gray and green, respectively. Important interacting residues and the NADPH/NADP ${ }^{+}$ cofactor (black carbons) are shown as sticks. Hydrogen bonds are indicated by brown dotted lines. While the orientation of $\mathbf{1 0}$ is rather similar in both DHFR variants, the compound tail is more solvent-exposed in TbDHFR: The PABA benzene and piperidine of $\mathbf{1 0}$ compete for interactions with Phe94 of TbDHFR, which thereby becomes exposed to the solvent. In hDHFR, the corresponding exposed residue is the polar Asn64 and the tail of 10 can interact with Phe31 deeper in the pocket, rendering the mode of binding more favorable in hDHFR. The results are presented for N1-deprotonated compounds, but similar observations were made with N1 protonation (Figure S6).

Compound $\mathbf{8}$ combined fragments of $\mathbf{3}$ and $\mathbf{7}$. Due mainly to the tail ester, this modification improved the inhibition for both TbPTR1 (IC $501 \mathrm{nM})$ and LmPTR1 (IC $500.1 \mu \mathrm{M})$. The activity against TbDHFR was similar to the N10-modified parent 3, whereas $L m D H F R$ and hDHFR inhibition were again influenced by the tail modification (IC 50 LmDHFR 8: $0.2 \mu \mathrm{M}$, 7: $0.1 \mu \mathrm{M}$; hDHFR 8: $13 \mu \mathrm{M}, 7: 12 \mu \mathrm{M})$. Compound 24 merged fragments of 3 and 23 . In TbPTR1, this boosted the nanomolar $\mathrm{IC}_{50}$ of $\mathbf{2 3}$ to the picomolar range, while the activity towards $L m P T R 1$ 
remained similar to 23 . This can be related to the N10 ethyl, which seems disfavored in LmPTR1 as judged by the modest inhibition of parent $3\left(\mathrm{IC}_{50} 13.3 \mu \mathrm{M}\right)$.

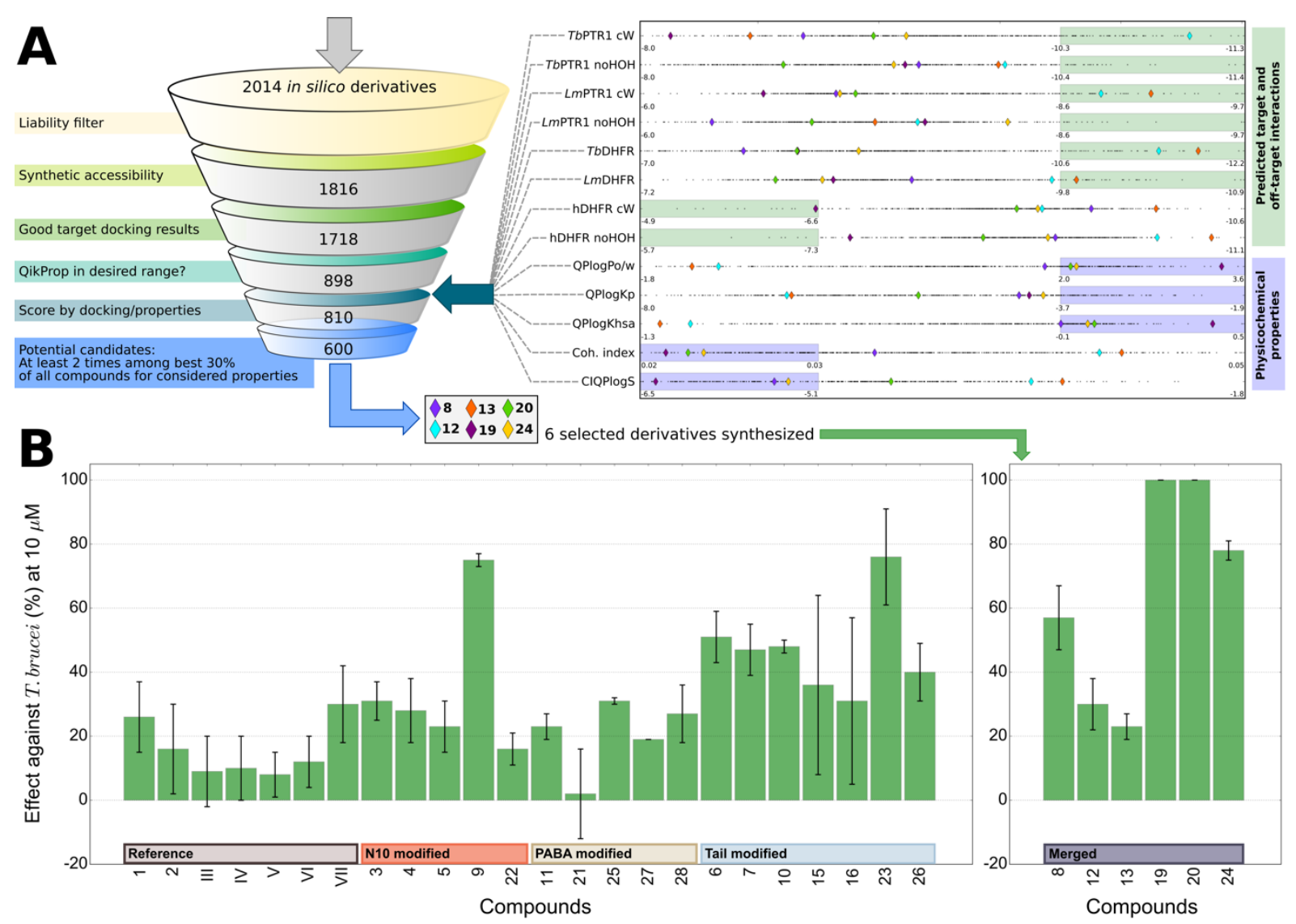

Figure 7. Workflow illustrating the prioritization of compounds in the in silico merged library (A) and anti-parasitic activity against $T$. brucei (B). (A) Compounds were filtered to reduce liabilities based on QikProp ${ }^{38}$ results and the synthetically least accessible compounds as predicted with the Ambit-SA tool ${ }^{39}$ were removed. As the next step, the compounds were filtered by the docking scores obtained for the different targets TbPTR1, LmPTR1, TbDHFR and $L m D H F R$, followed by evaluation of whether the QikProp properties that were found to correlate with antiparasitic activity (QPLogPo/w: octanol-water partition coefficient; QPlogKp: skin permeability; QPlogKhsa: binding to human serum albumin; coh. index: Index of cohesive interaction in solids, (no. of hydrogen bond acceptors x no. of hydrogen bond donors $\times 0.5$ / surface area ${ }^{40}$ and CIQPlogS: conformation-independent predicted aqueous solubility) were within the range typical for drug-like compounds. Finally, it was evaluated whether the compounds occurred at least twice among the best resulting $30 \%$ of compounds for each individual property, as indicated in the bar chart on the right-hand side by the green and blue regions for docking and QikProp results, respectively. All

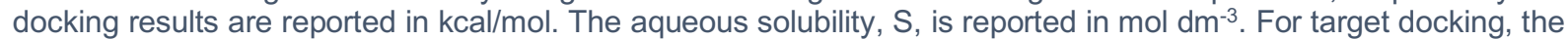
best docking scores and for off-target docking, the worst docking scores were considered favorable. QikProp results were evaluated based on the correlation with the anti-parasitic activity, with high values for correlating (QPlogPo/w, QPlogKp, QPlogKhsa) and low values for anti-correlating properties (coh. index, CIQPlogS). The bar plot (right) shows the range of the final set of 600 candidate compounds with black dots reporting the individual values for every property. 6 representatives were selected for synthesis in the merged series, such that they span the covered property range, as indicated by the colored diamonds. (B) Percentage inhibition against T. brucei brucei for reference compounds and members of the N10-, PABA and tail-modified series (left) and the selected representatives of the in silico merged library (right). The average of at least three independent determinations is shown with the standard deviation. The inactive compounds in the tail modified series, 14, 17 and 18 were omitted. Activities can be found in Table S7, SI.

Compounds 19 and 20 combined the scaffold of 16 with N10 modifications from 9 or $\mathbf{1}$, respectively. Both compounds were nanomolar inhibitors of both PTR1 variants. The parent compounds, 9 and $\mathbf{1}$, inhibited the parasite DHFR variants at micromolar to submicromolar levels, while 16 was inactive against all variants of DHFR. The combination with a favorable N10 substitution was able to restore medium micromolar anti-DHFR activity for the altered 
scaffold of parent 16 in the parasitic enzymes in 19 and 20. Thus, specific combinations of the N10 and tail modifications allow a fine-tuning of the target inhibition profile for enzymes of specific parasite species.

\section{The activity against $T$. brucei is related to the hydrophobicity of the compounds}

We determined the anti-parasitic effect on T. brucei brucei Lister 427 bloodstream forms and L. infantum intramacrophage amastigotes (Figure 7B and Table S7, SI). The pteridines were mostly inactive against $L$. infantum and, despite mostly being nanomolar inhibitors of TbPTR1 and micromolar inhibitors of TbDHFR, many of the new derivatives did not exceed $50 \% T$. brucei inhibition at $10 \mu \mathrm{M}$ compound concentration. Consistently, the multiple correlation coefficient between the TbPTR1 and TbDHFR IC 50 values and the T. brucei bloodstream form inhibition was $\mathrm{R}=0.35$ (equation $1, \mathrm{SI}$ ) and indicated that the levels of target enzyme inhibition were only weakly correlated with the exhibited anti-parasitic effect when assuming a linear correlation. PTR1 inhibition alone showed a Pearson correlation of 0.34 with the T. brucei inhibition, while $\mathrm{R}$ was only 0.24 for DHFR inhibition, possibly because all compounds considered are much stronger inhibitors of PTR1 than DHFR.

To consider other factors affecting the anti-parasitic activity of the compounds, we computed the physicochemical properties and ADMET predictors of the reference and N10, PABA and tail-modified series compounds and assessed property correlations with the measured effect on $T$. brucei, see Table 1. However, overall, only weak correlations of the individual properties with T. brucei inhibition were observed (Pearson R 0.47-0.55 and -0.41- -0.54; $\mathrm{R}^{2}$ 0.17-0.30; computed as defined in the SI).

The strongest correlation was found for the predicted skin permeability, log $\mathrm{Kp}$, as a descriptor linked to lipophilicity, with a Pearson $R$ of $0.55\left(R^{2} 0.30\right)$. The logPo/w and the binding to human serum albumin had slightly weaker correlations with the anti-parasitic effect (R: $0.49 R^{2}: 0.24$ and $R: 0.47 R^{2}: 0.22$, respectively). For these properties, an increase in the value corresponds with higher anti-T. brucei activity. In contrast, some properties showed anti-correlation, for instance the aqueous solubility and the cohesive index ${ }^{40}\left(R:-0.54 R^{2}: 0.29\right.$ and $R:-0.41 R^{2}$ : 0.17 , respectively). Taken together, all the data indicate an improved anti-parasitic effect with increased lipophilicity of the compounds.

We considered the correlating predicted properties as an additional prioritization filter for the in silico merged library, see Figure 7A. Of the six synthesized compounds, 19, 20 and 24 showed an improved percentage of $T$. brucei inhibition at $10 \mu \mathrm{M}$, as was suggested by their properties. For those compounds, $\mathrm{EC}_{50}$ values were determined, see Table 2. Indeed, the three more lipophilic compounds were found to have low micromolar $\mathrm{EC}_{50} \mathrm{~S}$ against $T$. brucei brucei with 24 being the best $\left(\mathrm{EC}_{50} 0.66 \pm 0.48 \mu \mathrm{M}\right)$ and they showed Sls of 3-38 on the basis of their cytotoxicity on THP-1 derived macrophages. 
Table 1. Descriptors with significant correlations with the observed effect on $T$. brucei for the reference compounds and pteridines of the N10-, PABA- and tail modified series calculated with QikProp. ${ }^{38}$ QPlogKp: Predicted skin permeability, log $\mathrm{K}_{\mathrm{p}}$; QPlogPo/w: Predicted octanol/water partition coefficient. QPlogKhsa: Prediction of binding to human serum albumin. Cohesive index: Index of cohesive interaction in solids, (no. of hydrogen bond acceptors $x$ no. of hydrogen bond donors x 0.5 / surface area $)^{40}$; CIQPlogS: Conformationindependent predicted aqueous solubility, $\log S$ with $S$ in $\mathrm{mol} \mathrm{dm}^{-3}$ being the concentration of the solute in a saturated solution that is in equilibrium with the crystalline solid. $\mathrm{R}$ (Pearson correlation) and $\mathrm{R}^{2}$ were calculated using the percentage of inhibition of the T. brucei brucei Lister 427 bloodstream form at $10 \mu \mathrm{M}$ compound concentration as defined in the SI. Only descriptors with at least a Pearson correlation/anti-correlation of 0.40/0.40 and two-tailed P-values lower than the chosen significance level $\alpha$ of 0.05 are reported. Covered range: property values obtained for the currently considered compounds. Recommended range: values the properties take for typical drug-like molecules. Resampling recovery rate indicates in how many cases (expressed as percentage) the same property was identified when leaving a single compound out from the data set. The optimization direction indicates whether higher or lower values would putatively lead to improved anti-parasitic effects.

\begin{tabular}{|c|c|c|c|c|c|c|c|c|c|c|c|}
\hline \multirow{2}{*}{$\begin{array}{l}\text { Predicted } \\
\text { property }\end{array}$} & \multirow[t]{2}{*}{$\mathbf{R}$} & \multirow[t]{2}{*}{$\mathbf{R}^{2}$} & \multirow[t]{2}{*}{$P$ value } & \multirow{2}{*}{$\begin{array}{c}\text { Resampling } \\
\text { recovery rate (\%) }\end{array}$} & \multicolumn{3}{|c|}{ Covered range } & \multicolumn{3}{|c|}{ Recommended range } & \multirow{2}{*}{$\begin{array}{l}\text { Optimization } \\
\text { direction }\end{array}$} \\
\hline & & & & & Min & to & $\max$ & Min & to & $\max$ & \\
\hline QPlogKp & 0.55 & 0.30 & 0.003 & 100 & -6.62 & - & -3.60 & -8.00 & - & -1.00 & $\uparrow$ \\
\hline QPlogPo/w & 0.49 & 0.24 & 0.010 & 96 & -1.02 & - & 2.92 & -2.00 & - & 6.50 & $\uparrow$ \\
\hline QPlogKhsa & 0.47 & 0.22 & 0.010 & 96 & -0.85 & - & 0.35 & -1.50 & - & 1.50 & $\uparrow$ \\
\hline Cohesive index & -0.41 & 0.17 & 0.040 & 56 & 0.02 & - & 0.04 & 0.00 & - & 0.05 & $\downarrow$ \\
\hline CIQPlogS & -0.54 & 0.29 & 0.004 & 96 & -6.71 & - & -3.19 & -6.50 & - & 0.50 & $\downarrow$ \\
\hline
\end{tabular}

Table 2. Properties with significant correlation with the observed effect on $T$. brucei for compounds in the merged series calculated with QikProp. ${ }^{38}$ The properties are defined as in Table 1. Values shown in bold face are within $90 \%$ of the previously determined top value or exceeded the previously obtained range for reference compounds and compounds in the N10-, PABA- and tail modified series, see Table 1. The activity against T. brucei brucei Lister 427 bloodstream form at $10 \mu \mathrm{M}$ compound concentration (\%inhibition) is given. For the most promising compounds, 19, 20 and 24, in addition, measured $\mathrm{EC}_{50}$ values, No-observed-adverse-effect level (NOAEL), and Selectivity indeces are reported. $\mathrm{EC}_{50}$ and NOAEL represent the arithmetic average of at least two independent measurements done in triplicate. NOAEL was determined by cytotoxicity assessment on THP-1-derived macrophages by a colorimetric MTT (3-(4,5-dimethylthiazol-2-yl)-2,5-diphenyl tetrazolium bromide) assay, as previously reported. ${ }^{41}$ N.D.: Not determined.

\begin{tabular}{|c|c|c|c|c|c|c|c|c|c|}
\hline Compound & $\begin{array}{c}\text { QP } \\
\operatorname{logKp}\end{array}$ & $\begin{array}{c}Q P \\
\log P o / w\end{array}$ & $\begin{array}{c}Q P \\
\text { logKhsa }\end{array}$ & $\begin{array}{l}\text { Cohesive } \\
\text { index }\end{array}$ & $\begin{array}{l}\text { CIQP } \\
\text { logS }\end{array}$ & $\begin{array}{c}\text { \%inhibition } \\
\text { of } T \text {. brucei } \\
\text { at } 10 \mu \mathrm{M} \pm \\
\text { SD }\end{array}$ & $\begin{array}{c}\mathrm{EC}_{50} T . \text { brucei } \\
{[\mu \mathrm{M}] \pm \mathrm{SD}}\end{array}$ & $\begin{array}{c}\mathrm{CC}_{50} \text { or } \\
\text { NOAEL }[\mu \mathrm{M}]\end{array}$ & $\begin{array}{l}\text { Selectivity } \\
\text { index } \\
\left(\mathrm{CC}_{50} \text { or }\right. \\
\text { lower limit } \\
\left.\text { / } \mathrm{EC}_{50}\right)\end{array}$ \\
\hline 8 & -5.18 & 2.02 & 0.04 & 0.03 & -5.32 & $57 \pm 10$ & N.D. & N.D. & N.D. \\
\hline 12 & -6.74 & -1.16 & 0.05 & 0.04 & -4.53 & $30 \pm 8$ & N.D. & N.D. & N.D. \\
\hline 13 & -6.48 & -1.32 & 0.43 & 0.04 & -6.35 & $23 \pm 4$ & N.D. & N.D. & N.D. \\
\hline 19 & -4.60 & 3.36 & -1.23 & 0.02 & -3.20 & $100 \pm 0$ & $4.53 \pm 0.42$ & $\begin{array}{c}12.5<\mathrm{CC}_{50}< \\
25\end{array}$ & 3 \\
\hline 20 & -5.16 & 2.09 & -1.14 & 0.02 & -3.44 & $100 \pm 0$ & $1.30 \pm 0.05$ & $\begin{array}{c}12.5<\mathrm{CC}_{50}< \\
25\end{array}$ & 10 \\
\hline 24 & -3.91 & 2.19 & 0.07 & 0.02 & -5.43 & $78 \pm 3$ & $0.66 \pm 0.48$ & $25<\mathrm{CC}_{50}<50$ & 38 \\
\hline Pentamidine & N.D. & N.D. & N.D. & N.D. & N.D. & N.D. & $0.0019 \pm 0.0005$ & 10 & 5263 \\
\hline
\end{tabular}




\section{Increased hydrophobicity often leads to liabilities}

Potential liabilities were assessed by determining the inhibition of the $h E R G$ potassium channel, five isoforms of CYP450 (1A2, 2C9, 2C19, 2D6 and 3A4), cytotoxicity against A459 cells (human lung adenocarcinoma epithelial cell line) and mitochondrial toxiticity against 786 O cells (renal carcinoma cell line) for all compounds at a concentration of $10 \mu \mathrm{M}$. The results are shown in Figure 8. Further, the compounds passed a check for Pan-assay interference compounds (PAINS).

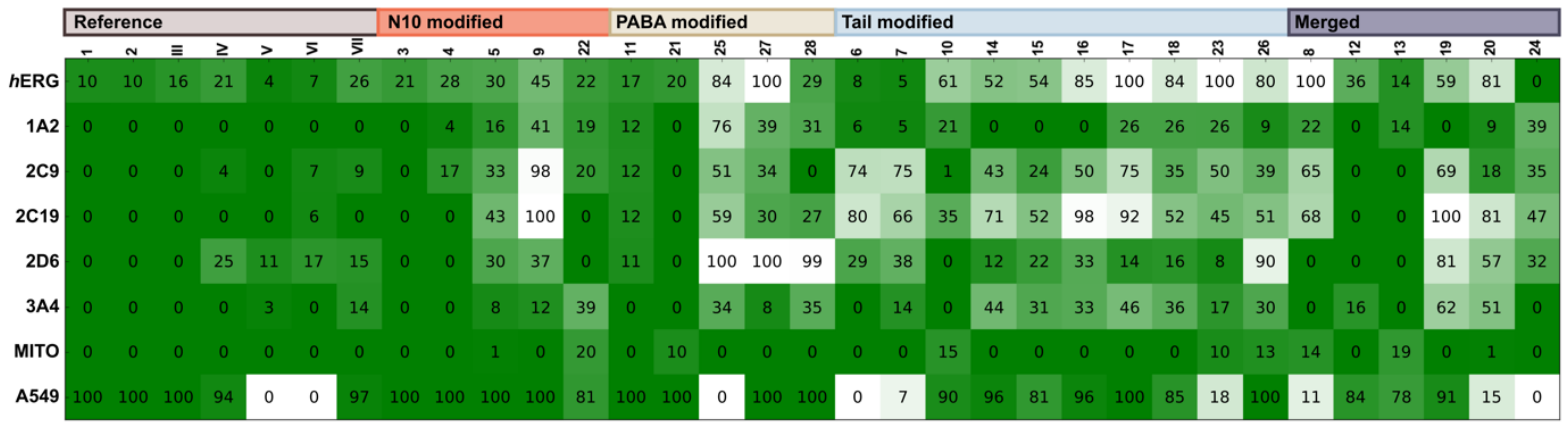

Figure 8. Heatmap representation of the liability assessment results. Inhibition of $h$ ERG, five CYP isoforms (1A2, 2C9, 2C19, 2D6 and 3A4), mitochondrial toxicity (MITO) and growth inhibition of A549 cells were determined at 10 $\mu \mathrm{M}$. The data are represented as percentages on a color scale from green (desired) to white (undesired value) with values reported in the map. For the inhibitory activities against $h$ ERG, CYP isoforms and mitochondrial toxicity: green $=0 \%$, white $=100 \%$ inhibition/toxicity, while for A549 cell growth inhibition green $=100 \%$ and white $=0 \%$ growth. The values are reported in Tables S8,S9.

The reference compounds and the N10 series largely exhibited a safe profile. Aromatic modifications to the compound tail region, for instance in 27, 17 and 20 (PABA, Tail and Merged series, respectively) were associated with notable $h E R G$ liabilities. Increasing the hydrophobicity of the compounds further led to liabilities against some CYP isoforms, in particular, 2C9 and 2C19. The shortened tails of $\mathbf{2 5}$ and 27 resulted in a strong effect on CYP isoform 2D6. Finally, several of the bulky, more hydrophobic compounds resulted in a cytostatic or cytotoxic effect on A549 cells, thus demonstrating a need for further optimization with increasing hydrophobicity being associated with greater liabilities.

In line with these observations, two of the best inhibitors of $T$. brucei bloodstream forms, 19 and 20 show $54 \%$ and $81 \% h E R G$ inhibition, respectively. 19 and, in many cases also 20 , also affected various CYP isoforms: 2C19 was inhibited to $100 \%$ and $81 \%, 2 \mathrm{D} 6$ to $81 \%$ and $57 \%$ and $3 \mathrm{~A} 4$ to $62 \%$ and $51 \%$, respectively. 19 further showed $69 \%$ CYP 2 C9 inhibition. Finally, $\mathbf{2 0}$ was cytostatic with A549 cell growth reduced to $15 \%$ and $\mathbf{2 4}$ showed cytotoxicity, effectively completely inhibiting cell growth. Taken together, the most efficient inhibitors of $T$. brucei bloodstream forms were found to suffer from liabilities associated with their large hydrophobicity and require careful optimization of their cellular specificity. 


\section{Discussion}

Applying a multitarget-based approach to the development of novel therapies for HAT and leishmaniasis, we here focused on pteridine-based inhibitors of $L$. major and T. brucei PTR1 and DHFR and successfully designed the first known picomolar inhibitors of TbPTR1. While LmPTR1/LmDHFR inhibition was previously explored for this compound class, we here demonstrated the potential of pteridine-based inhibitors against the TbPTR1/TbDHFR system. ${ }^{26}$ We solved a crystal structure of the reference compound 1 bound to TbPTR1 to confirm the overlap in observed binding modes between the two PTR1 variants and the preference of the inhibitor-like bound orientation in TbPTR1. Guided by our detailed comparative study of on- and off-targets in the parasitic and human folate pathway ${ }^{31}$, crystallographic reference structures and enzymatic evaluation of published reference pteridine ${ }^{26,30}$, we designed 26 new pteridine derivatives that mostly have improved activity and selectivity. For their synthesis, we made use of an advanced MW-assisted protocol to improve the reaction yield of the pteroid step with reduced reaction time. Further determination of crystal structures of complexes and computational docking enabled us to obtain a complete characterization of the binding modes of the pteridines to their molecular targets and supported the derivation of a SAR.

The compounds were also tested against the human off-targets hDHFR and hTS. While they were sometimes only modestly selective for the parasitic DHFR variant, many showed 1000fold and higher selectivities for PTR1 over the off-targets and thus, the novel PTR1 inhibitors can overall be considered selective for the parasite proteins.

While many compounds exhibited excellent inhibitory activity at the target level, they were often only modest inhibitors of $T$. brucei brucei bloodstream forms and inactive towards $L$. infantum intracellular amastigotes in vitro. We found that increased lipophilicity correlated with improved effects on T. brucei. We were able to prioritize compounds from an in silico library for synthesis by using predicted ADMET-related properties which suggested a likely improvement of the trypanocidal effect. In this way, we found three improved compounds,19, 20 and 24, with low micromolar inhibition of $T$. brucei brucei $\left(\mathrm{EC}_{50} 0.66-4.53 \mu \mathrm{M}\right)$. The result demonstrates that the approach employed here of combining property prediction correlation with multitarget-based compound design is a valuable approach to informing compound design, even when anti-parasitic data are available only as a percentage of inhibition determined at a single compound concentration. Further, integration of transport related considerations in the design ${ }^{31}$, or using, for instance, structurally related scaffolds reported in the literature, which show inhibition of the Leishmania parasite, and a similar property-based correlation concept to that presented here, may help to overcome the current limitations of the pteridine-based compounds as inhibitors of intracellular parasites. Our data show that, overall, optimization for increased lipophilicity leads to more potent pteridine-based $T$. brucei inhibitors. 
However, increased lipophilicity can also introduce compound liabilities, e.g. for $h E R G$ and CYPs. Strategies to avoid these, for instance by making use of a similar property-based optimization strategy, should thus be incorporated in future design efforts.

\section{Experimental Procedures}

\section{Resource Availability}

Lead Contact: Further information and requests for resources and reagents should be directed to and will be fulfilled by the Lead Contact, Rebecca Wade (Rebecca.wade@h-its.org)

Materials Availability: All unique/stable reagents generated in this study will be made available without restriction.

Data and Code Availability: Crystal structures described in this paper are available in the Protein Data Bank with identifiers: 6rx5 (TbPTR1-NADPH/NADP ${ }^{+}-1$ ), 6rx0 (TbPTR1NADPH/NADP $\left.{ }^{+}-3\right)$, 6rx6 (TbPTR1-NADPH/NADP $\left.{ }^{+}-4\right)$, 6rxc (LmPTR1-NADPH/NADP $\left.{ }^{+}-1\right)$.

Other datasets generated during this study are available at FairdomHub:

https://fairdomhub.org/investigations/417

The detailed synthesis methodology for compounds 1-28, with the reductive alkylation of amines using nitriles, amide coupling reactions, microwave alkylation and $S_{N} A r$ reactions for the preparation of 4-substituted benzaldehyde, is reported in the SI. The structures of the isolated compounds were confirmed by NMR spectroscopy and mass spectrometry and purity was determined by elemental analyses and melting point interval measurement. Liquid chromatography-mass spectrometry (LC-MS) was used to obtain the molecular mass and analyze product purity.

Recombinant proteins for enzymatic assays and crystallization were expressed and purified as reported previously. ${ }^{26,41,42}$ Crystals of TbPTR1 and LmPTR1 were prepared as described elsewhere and PTR1-cofactor-inhibitor ternary complexes were obtained by soaking. ${ }^{37,41}$ Data collection and refinement statistics are reported in Tables S2 and S3. The structures were solved by molecular replacement using a TbPTR1 (PDB-ID 5jdc) or LmPTR1 tetramer (PDBID $5 \mid 4 n$ ) as the search model. ${ }^{37,41}$ Coordinates and structure factors have been deposited in the RCSB under the PDB-IDs 6rx5 (TbPTR1-NADPH/NADP $\left.{ }^{+}-1\right)$, 6rx0 (TbPTR1NADPH/NADP $\left.{ }^{+}-3\right)$, 6rx6 (TbPTR1-NADPH/NADP $\left.{ }^{+}-4\right)$, and 6rxc (LmPTR1-NADPH/NADP $\left.{ }^{+}-4\right)$. In vitro assessment of enzyme inhibition was carried out as previously reported. ${ }^{41,43,44}$

Schrödinger software was used for ligand (LigPrep) and receptor (PrepWizard) preparation. ${ }^{45}$

Receptors were based on the following PDB-IDs/structures: TbPTR1: 2x9g, LmPTR1: 1e92, TbDHFR: 3rg9, hDHFR: 1u72, and LmDHFR: our previously published homology model. ${ }^{31}$ Conserved structural water sites in PTR1 and hDHFR were identified by WatCH clustering. ${ }^{46}$ SiteMap was used to compute the pocket volumes of DHFR models. ${ }^{45}$ Docking studies were 
performed using Schrödinger Glide in standard precision (SP) and extra precision (XP) or with the Induced Fit (IF) protocol to allow for refinement of binding site residues. ${ }^{45}$ Validation results for the methodology are reported in Tables S10-S12. Physicochemical descriptors and ADMET parameters were computed using QikProp. ${ }^{38}$ Python scripts were employed for the correlation analysis. Checks for PAINS, undesirable substructure moieties, covalent inhibition and compliance with the rule-of-five were performed with the FAFdrugs 4 webserver. ${ }^{47}$

All compounds were evaluated in vitro against T. brucei brucei Lister 427 bloodstream forms in a modified resazurin-based assay and against $L$. infantum intracellular amastigotes as described previously. ${ }^{48,49}$ To assess potential compound liabilities, $h E R G$ cardiotoxicity, inhibition of the CYP450 isoforms 1A2, 2C9, 2C19, 2D6 and 3A4, mitochondrial toxicity and cytotoxicity against A549 cells were assayed as described previously. ${ }^{41}$ Further details of all experimental procedures are given in the SI.

\section{Supplemental Information}

Supplemental Figures S1-8, Supplemental Tables S1-12, Supplemental experimental procedures and compound characterization.

Additional supplementary data at https://fairdomhub.org/investigations/417:

QikProp prediction results for synthesized and in silico pteridines and corresponding SOP. PAINS filtering results, Python modules for correlating QikProp data with experimental activities and for computing a multiple correlation between target and parasite inhibition. Compound library construction data and SOP, prepared docking receptors (PDB) with SOP, all Glide XP rigid-body docking results and SOP as well as selected discussed induced fit docking results and corresponding SOP.

\section{Acknowledgements}

This work has received funding from the European Union's Seventh Framework Programme for research, technological development, and demonstration under grant agreement no. 603240 (NMTrypl, New Medicines for Trypanosomatidic Infections, https://fp7-nmtrypi.eu/). We thank Prof. Antonio Carta, University of Sassari, for providing the reference compounds III-VII. I.P., J.P.-H. and R.C.W. gratefully acknowledge the support of the Klaus Tschira Foundation. J.P.-H. acknowledges support from the Polish National Science Centre (grant no. 2016/21/D/NZ1/02806) and the BIOMS program at the Interdisciplinary Center for Scientific Computing (IWR), Heidelberg University. 


\section{Author Contributions}

Conceptualization, M.P.C., A.V., R.C.W.; Computational Methodology and Investigation, I.P., J. P.-H.; Chemical synthesis Methodology and Investigation, A.Q.; Enzyme assay Methodology and Investigation, R.L., M.S., P.L.; Crystallography Methodology and Investigation, G.L., F.D.P., L.D.I., C.P.; ADMET Methodology and Investigation, S.G., G.W., B.E., M.K.; Parasite assay Methodology and Investigation, N.S.; Writing - Original Draft, I.P., Writing - Review \& Editing, I.P., M.P.C., R.C.W.; Supervision, S.M., A.C.S., M.P.C, A.V., R.C.W.

\section{Declaration of interests}

The authors declare no competing interests.

\section{References}

1. Neglected tropical diseases. https://www.who.int/neglected diseases/en/; Accessed May 2020.

2. The WHO Strategic and Technical Advisory Group for Neglected Tropical Diseases (WHO STAG). https://www.who.int/neglected diseases/diseases/Adoption additional NTDs.pdf?ua=1, $\% 202017$.

3. Barrett, M. P., Burchmore, R. J. S., Stich, A., Lazzari, J. O., Frasch, A. C., Cazzulo, J. J. and Krishna, S. (2003). The trypanosomiases. Lancet. 362, 1469-1480.

4. Blum, J., Schmid, C. and Burri, C. (2006). Clinical aspects of 2541 patients with second stage human African trypanosomiasis. Acta Trop. 97, 55-64.

5. Stuart, K., Brun, R., Croft, S., Fairlamb, A., Gürtler, R. E., McKerrow, J., Reed, S. and Tarleton, R. (2008). Kinetoplastids: related protozoan pathogens, different diseases. J. Clin. Invest. 118, 1301-1310.

6. Cunningham, A. C. (2002). Parasitic adaptive mechanisms in infection by Leishmania. Exp. Mol. Pathol. 72, 132-141.

7. Herwaldt, B. L. (1999). Leishmaniasis. Lancet. 354, 1191-1199.

8. Castillo, E., Dea-Ayuela, M. A., Bolás-Fernández, F., Rangel, M. and González-Rosende, M. E. (2010). The kinetoplastid chemotherapy revisited: current drugs, recent advances and future perspectives. Curr. Med. Chem. 17, 4027-4051.

9. Machado-Silva, A., Goulart Guimarães, P. P., Pereira Tavares, C. A. and Sinisterra, R. D. (2015). New perspectives for leishmaniasis chemotherapy over current anti-leishmanial drugs: a patent landscape. Expert Opin. Ther. Pat. 25, 247-260.

10. Gilbert, I. H. (2013). Drug discovery for neglected diseases: molecular target-based and phenotypic approaches. J. Med. Chem. 56, 7719-7726.

11. Müller, J. and Hemphill, A. (2016). Drug target identification in protozoan parasites. Expert Opin. Drug Discov. 11, 815-824.

12. Borsari, C., Quotadamo, A., Ferrari, S., Venturelli, A., Cordeiro-da-Silva, A., Santarem, N. and Costi, M. P. (2018). Chapter Two - Scaffolds and Biological Targets Avenue to Fight Against Drug Resistance in Leishmaniasis. Annu. Rep. Med. Chem. 51, 39-95.

13. Shuvalov, O., Petukhov, A., Daks, A., Fedorova, O., Vasileva, E. and Barlev, N. A. (2017). One-carbon metabolism and nucleotide biosynthesis as attractive targets for anticancer therapy. Oncotarget 8, 23955-23977.

14. Anderson, A. C. and Wright, D. L. (2014). Antifolate agents: a patent review (2010 - 2013). Expert Opin. Ther. Pat. 24, 687-697.

15. Hawser, S., Lociuro, S. and Islam, K. (2006). Dihydrofolate reductase inhibitors as 
antibacterial agents. Biochem. Pharmacol. 71, 941-948.

16. Yuthavong, Y., Yuvaniyama, J., Chitnumsub, P., Vanichtanankul, J., Chusacultanachai, S., Tarnchompoo, B., Vilaivan, T. and Kamchonwongpaisan, S. (2005). Malarial (Plasmodium falciparum) dihydrofolate reductase-thymidylate synthase: structural basis for antifolate resistance and development of effective inhibitors. Parasitology 130, 249-259.

17. Christensen, K. E. and MacKenzie, R. E. (2006). Mitochondrial one-carbon metabolism is adapted to the specific needs of yeast, plants and mammals. Bioessays 28, 595-605.

18. Cullia, G., Tamborini, L., Conti, P., De Micheli, C. and Pinto, A. (2018). Folates in Trypanosoma brucei: Achievements and Opportunities. ChemMedChem 13, 2150-2158.

19. Bello, A. R., Nare, B., Freedman, D., Hardy, L. and Beverley, S. M. (1994). PTR1: A reductase mediating salvage of oxidized pteridines and methotrexate resistance in the protozoan parasite Leishmania major. Proc. Natl. Acad. Sci. U. S. A. 91, 11442-11446.

20. Dawson, A., Gibellini, F., Sienkiewicz, N., Tulloch, L. B., Fyfe, P. K., McLuskey, K., Fairlamb, A. H. and Hunter, W. N. (2006). Structure and reactivity of Trypanosoma brucei pteridine reductase: inhibition by the archetypal antifolate methotrexate. Mol. Microbiol. 61, 1457-1468.

21. Vickers, T. J. and Beverley, S. M. (2011). Folate metabolic pathways in Leishmania. Essays Biochem. 51, 63-80.

22. Ong, H. B., Sienkiewicz, N., Wyllie, S. and Fairlamb, A. H. (2011). Dissecting the Metabolic Roles of Pteridine Reductase 1 in Trypanosoma brucei and Leishmania major. J. Biol. Chem. 286, 10429-10438.

23. Sienkiewicz, N., Ong, H. B. and Fairlamb, A. H. (2010). Trypanosoma brucei pteridine reductase 1 is essential for survival in vitro and for virulence in mice. Mol. Microbiol. 77, 658-671.

24. Mpamhanga, C. P., Spinks, D., Tulloch, L. B., Shanks, E. J., Robinson, D. A., Collie, I. T., Fairlamb, A. H., Wyatt, P. G., Frearson, J. A., Hunter, W. N., Gilbert, I. H. and Brenk, R. (2009). One Scaffold, Three Binding Modes: Novel and Selective Pteridine Reductase 1 Inhibitors Derived from Fragment Hits Discovered by Virtual Screening. J. Med. Chem. 52, 4454-4465.

25. Spinks, D., Ong, H. B., Mpamhanga, C. P., Shanks, E. J., Robinson, D. A., Collie, I. T., Read, K. D., Frearson, J. A., Wyatt, P. G., Brenk, R., Fairlamb, A. H. and Gilbert, I. H. (2011). Design, Synthesis and Biological Evaluation of Novel Inhibitors of Trypanosoma brucei Pteridine Reductase 1. ChemMedChem 6, 302-308.

26. Cavazzuti, A., Paglietti, G., Hunter, W. N., Gamarro, F., Piras, S., Loriga, M., Allecca, S., Corona, P., McLuskey, K., Tulloch, L., Gibellini, F., Ferrari, S. and Costi, M. P. (2008). Discovery of potent pteridine reductase inhibitors to guide antiparasite drug development. Proc. Natl. Acad. Sci. U. S. A. 105, 1448-1453.

27. Ivanetich, K. M. and Santi, D. V. (1990). Bifunctional thymidylate synthase-dihydrofolate reductase in protozoa. FASEB J. 4, 1591-1597.

28. Schormann, N., Senkovich, O., Walker, K., Wright, D. L., Anderson, A. C., Rosowsky, A., Ananthan, S., Shinkre, B., Velu, S. and Chattopadhyay, D. (2008). Structure-based approach to pharmacophore identification, in silico screening, and three-dimensional quantitative structure-activity relationship studies for inhibitors of Trypanosoma cruzi dihydrofolate reductase function. Proteins 73, 889-901.

29. Schormann, N., Velu, S. E., Murugesan, S., Senkovich, O., Walker, K., Chenna, B. C., Shinkre, B., Desai, A. and Chattopadhyay, D. (2010). Synthesis and characterization of potent inhibitors of Trypanosoma cruzi dihydrofolate reductase. Bioorg. Med. Chem. 18, 4056-4066.

30. Corona, P., Gibellini, F., Cavalli, A., Saxena, P., Carta, A., Loriga, M., Luciani, R., Paglietti, G., Guerrieri, D., Nerini, E., Gupta, S., Hannaert, V., Michels, P. A. M., Ferrari, S. and Costi, P. M. (2012). Structure-based selectivity optimization of piperidine-pteridine derivatives as potent Leishmania pteridine reductase inhibitors. J. Med. Chem. 55, 8318-8329.

31. Panecka-Hofman, J., Pöhner, I., Spyrakis, F., Zeppelin, T., Di Pisa, F., Dello lacono, L., Bonucci, A., Quotadamo, A., Venturelli, A., Mangani, S., Costi, M. P. and Wade, R. C. (2017). Comparative mapping of on-targets and off-targets for the discovery of anti- 
trypanosomatid folate pathway inhibitors. Biochim. Biophys. Acta, Gen. Subj. 1861, 32153230.

32. Tulloch, L. B., Martini, V. P., lulek, J., Huggan, J. K., Lee, J. H., Gibson, C. L., Smith, T. K., Suckling, C. J. and Hunter, W. N. (2010). Structure-Based Design of Pteridine Reductase Inhibitors Targeting African Sleeping Sickness and the Leishmaniases. J. Med. Chem. 53, 221-229.

33. Quotadamo, A., Linciano, P., Costi, M. P. and Venturelli, A. (2019). Optimization of Nalkylation in the Synthesis of Methotrexate and Pteridine-based Derivatives Under Microwave-Irradiation. ChemistrySelect 4, 4429-4433.

34. Sajiki, H., Ikawa, T. and Hirota, K. (2004). Reductive and Catalytic Monoalkylation of Primary Amines Using Nitriles as an Alkylating Reagent. Org. Lett. 6, 4977-4980.

35. Ikawa, T., Fujita, Y., Mizusaki, T., Betsuin, S., Takamatsu, H., Maegawa, T., Monguchia, Y. and Sajiki, H. (2012). Selective N-alkylation of amines using nitriles under hydrogenation conditions: facile synthesis of secondary and tertiary amines. Org. Biomol. Chem. 10, 293304.

36. Gourley, D. G., Schüttelkopf, A. W., Leonard, G. A., Luba, J., Hardy, L. W., Beverley, S. M. and Hunter, W. N. (2001). Pteridine reductase mechanism correlates pterin metabolism with drug resistance in trypanosomatid parasites. Nat. Struct. Biol. 8, 521-525.

37. Di Pisa, F., Landi, G., Dello lacono, L., Pozzi, C., Borsari, C., Ferrari, S., Santucci, M., Santarem, N., Cordeiro-da-Silva, A., Moraes, C. B., Alcantara, L. M., Fontana, V., FreitasJunior, L. H., Gul, S., Kuzikov, M., Behrens, B., Pöhner, I., Wade, R. C., Costi, M. P. and Mangani, S. (2017). Chroman-4-One Derivatives Targeting Pteridine Reductase 1 and Showing Anti-Parasitic Activity. Molecules 22, 426.

38. Schrödinger, LLC, New York, NY, Schrödinger Release 2015-4, 2015, QikProp v4.6.

39. AMBIT. (2018). Ambit-SA (SyntheticAccessibilityCli), http://ambit.sourceforge.net/reactor.html.

40. Jorgensen, W. L. and Duffy, E. M. (2000). Prediction of drug solubility from Monte Carlo simulations. Bioorg. Med. Chem. Lett. 10, 1155-1158. [42]

41. Borsari, C., Luciani, R., Pozzi, C., Poehner, I., Henrich, S., Trande, M., Cordeiro-da-Silva, A., Santarem, N., Baptista, C., Tait, A., Di Pisa, F., Dello lacono, L., Landi, G., Gul, S., Wolf, M., Kuzikov, M., Ellinger, B., Reinshagen, J., Witt, G., Gribbon, P., Kohler, M., Keminer, O., Behrens, B., Costantino, L., Tejera Nevado, P., Bifeld, E., Eick, J., Clos, J., Torrado, J., Jiménez-Antón, M. D., Corral, M. J., Alunda, J. M., Pellati, F., Wade, R. C., Ferrari, S., Mangani, S. and Costi, M. P. (2016). J. Med. Chem. 59, 7598-7616. [33]

42. Cardinale, D., Guaitoli, G., Tondi, D., Luciani, R., Henrich, S., Salo-Ahen, O. M., Ferrari, S., Marverti, G., Guerrieri, D., Ligabue, A., Frassineti, C., Pozzi, C., Mangani, S., Fessas, D., Guerrini, R., Ponterini, G., Wade, R. C. and Costi, M. P. (2011). Protein-protein interface-binding peptides inhibit the cancer therapy target human thymidylate synthase. Proc. Natl. Acad. Sci. U. S. A. 108, E542-E549.

43. Ferrari, S., Morandi, F., Motiejunas, D., Nerini, E., Henrich, S., Luciani, R., Venturelli, A., Lazzari, S., Calò, S., Gupta, S., Hannaert, V., Michels, P. A. M., Wade, R. C. and Costi, M. P. (2011). Virtual Screening Identification of Nonfolate Compounds, Including a CNS Drug, as Antiparasitic Agents Inhibiting Pteridine Reductase. J. Med. Chem. 54, 211-221.

44. Tan, X., Huang, S., Ratnam, M., Thompson, P. D. and Freisheim, J. H. (1990). The Importance of Loop Region Residues $40-46$ in Human Dihydrofolate Reductase as Revealed by Site-directed Mutagenesis. J. Biol. Chem. 265, 8027-8032.

45. Schrödinger, LLC, New York, NY, Schrödinger Release 2015-4, 2015, LigPrep v3.6; Epik v3.4; Protein Preparation Wizard (PrepWizard); SiteMap v3.7; Glide v6.9; Induced Fit Docking Protocol, Prime v4.2.

46. Sanschagrin, P. C. and Kuhn, L. A. (1998). Cluster analysis of consensus water sites in thrombin and trypsin shows conservation between serine proteases and contributions to ligand specificity. Protein Sci. 7, 2054-2064.

47. Lagorce, D., Sperandio, O., Baell, J. B., Miteva, M. A. and Villoutreix, B. O. (2015). FAFDrugs3: a web server for compound property calculation and chemical library design. Nucleic Acids Res. 43, W200-207. https://fafdrugs4.mti.univ-paris-diderot.fr. 
48. Bowling, T., Mercer, L., Don, R., Jacobs, R. and Nare, B. (2012). Application of a resazurinbased high-throughput screening assay for the identification and progression of new treatments for human African trypanosomiasis. Int. J. Parasitol. Drugs Drug Resist. 2, 262 270.

49. Sereno, D., Cavaleyra, M., Zemzoumi, K., Maquaire, S., Ouaissi, A. and Lemesre, J. L. (1998). Axenically Grown Amastigotes of Leishmania infantum Used as an In Vitro Model To Investigate the Pentavalent Antimony Mode of Action. Antimicrob. Agents Chemother. 42, 3097-3102. 